13

\title{
Удельное электросопротивление тонких углеродных пленок с различной долей $s p$-связей
}

\author{
(С) И.А. Завидовский, О.А. Стрелецкий, О.Ю. Нищак, А.А. Хайдаров, А.В. Павликов \\ Московский государственный университет им. М.В. Ломоносова, фризический фракультет, \\ 119991 Москва, Россия \\ e-mail: ia.zavidovskii@physics.msu.ru
}

Поступило в Редакцию 31 декабря 2018 г.

В окончательной редакции 30 мая 2019 г.

Принято к публикации 30 мая 2019 г.

Методом ионно-плазменного импульсно-дугового распыления графита в атмосфере метана были получены углеродные пленки с различной долей $s$-связей. Данные спектроскопии комбинационного рассеяния света и просвечивающей электронной микроскопии свидетельствуют о том, что с ростом концентрации метана в рабочем объеме увеличивается содержание фазы, соответствующей углеродным цепочкам с $s p$-гибридизацией. Электросопротивление образцов хорошо коррелирует с долей $s p$-гибридизованного углерода в структуре исследуемых пленок.

Ключевые слова: $s$-углерод, аморфный углерод, КР-спектроскопия, электронная дифракция, просвечивающая электронная микроскопия.

DOI: $10.21883 /$ JTF.2020.01.48677.453-18

\section{Введение}

Тонкие пленки, такие как a-C и ta-C (состоящие из аморфного углерода с преобладающей долей $s p^{2}$-связей и $s p^{3}$-связей соответственно [1]), представляют большой интерес, так как могут применяться в качестве защитных покрытий [2], элементов наноэлектромеханических систем [3], мемристоров [4], для оптических приложений [5] и в других областях. Изменяя параметры получения углеродных пленок, можно управлять их фазовым составом, что позволяет в значительной мере влиять на конечные физические свойства [5].

Одной из причин внимания ученых к синтезу и исследованию наноразмерных углеродных материалов являются характерные для таких структур особенности электронного транспорта. В частности, в материалах, изготавливаемых на основе графена и углеродных нанотрубок, наблюдается баллистическая проводимость [6,7]. Появление этого эффекта предсказано в $s p$-гибридизованных углеродных цепочках [8]. $S p$-углерод интересен также тем, что его электрофизические свойства варьируются в зависимости от продольного растяжения [9] и количества атомов в цепочке [10].

В настоящей работе получение углеродных пленок со структурой типа ta-C, содержащих $s p$-гибридизованную фазу, производилось методом ионно-плазменного импульсно-дугового распыления графита. Данный метод позволяет, регулируя параметры осаждения, такие как остаточное давление и состав рабочего газа, изменять в широком спектре свойства получаемых покрытий, управляя как их морфологией, так и фазовым составом.

В данной статье приводятся результаты исследования структурных свойств и удельного электросопротивления полученных углеродных покрытий в зависимости от условий их нанесения.

\section{1. Получение образцов и методы их исследования}

Углеродные пленки изготавливались методом ионноплазменного импульсно-дугового распыления графитового катода при мощности разряда $1 \mathrm{~kW}$ в среде смеси газов $\mathrm{Ar}$ и $\mathrm{CH}_{4}$ в высоковакуумной камере с предварительной безмасляной откачкой до давления $10^{-4}$ Torr. В качестве подложек использовались пластины $\mathrm{Si}$, Ti и монокристаллический скол $\mathrm{NaCl}$. Перед началом процесса напыления осуществлялась очистка подложек путем травления ионами Ar с энергией $1 \mathrm{keV}$.

Напыление углеродных пленок производилось при рабочем давлении в камере $10^{-3}$ Torr, при этом процентное соотношение метана в смеси газов варьировалось от 0 до $52 \%$. Время импульса разряда составляло порядка 1 $\mathrm{ms}$, частота следования импульсов $-1 \mathrm{~Hz}$, температура поверхности подложек не превышала $30^{\circ} \mathrm{C}$. Толщина покрытия, наносимого за один импульс, составляла порядка $0.5 \mathrm{~nm}$.

Структура и фазовый состав образцов исследовались методами спектроскопии комбинационного рассеяния (КР-спектроскопии) и просвечивающей электронной микроскопии (ПЭМ).

КР-спектроскопия проводилась на спектрометре Horiba HR 800 с длиной волны гелий-неонового лазера $632.8 \mathrm{~nm}$, мощностью $1 \mathrm{~mW}$ и разрешающей способностью $1 \mathrm{~cm}^{-1}$. Исследования с помощью просвечивающей электронной микроскопии осуществлялись на базе просвечивающего электронного микроскопа LEO 912 ab. 


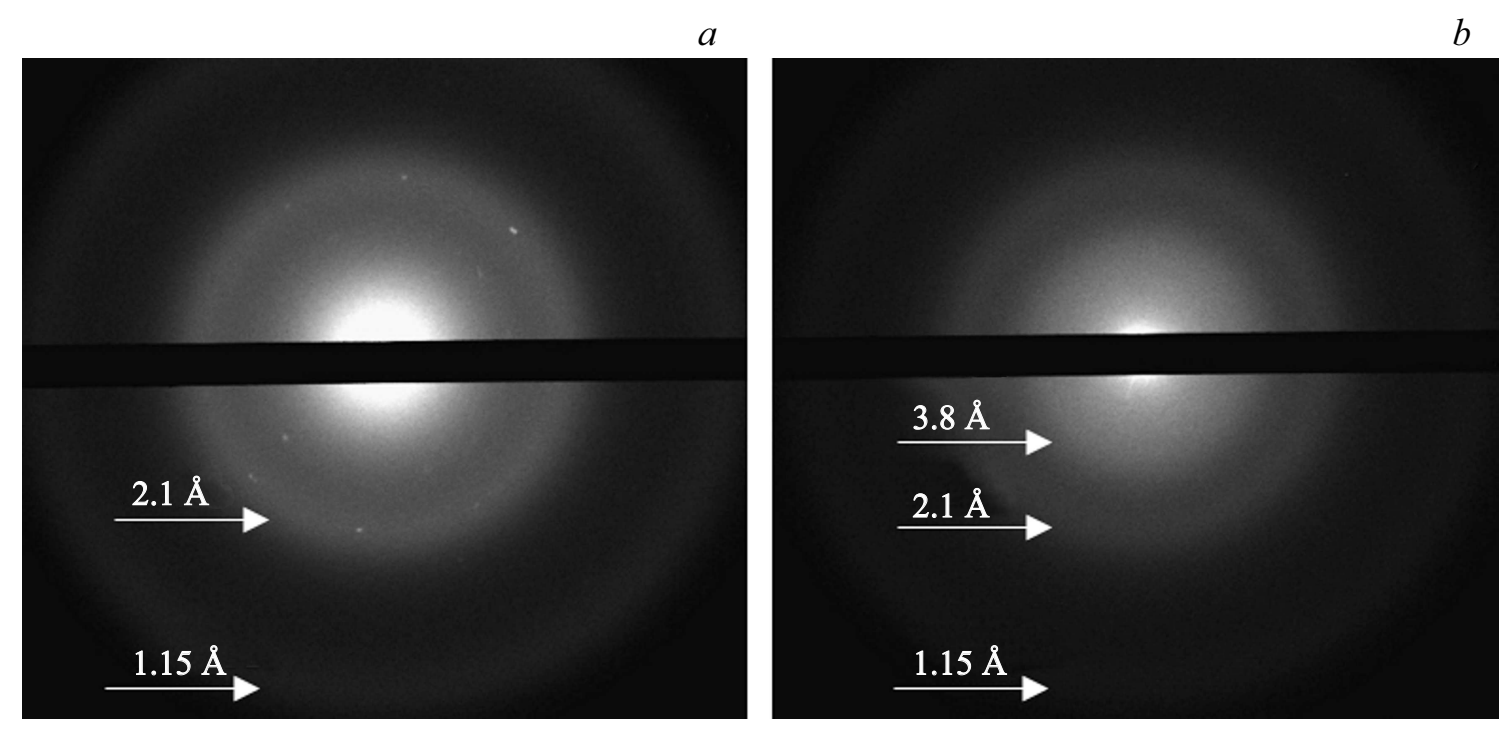

Рис. 1. Дифракционные картины образцов, полученных в смеси газов аргона и метана: $a-0 \% \mathrm{CH}_{4}, b-52 \% \mathrm{CH}_{4}$.

Удельное электросопротивление измерялось при помощи системы четырехточечного зондового измерения Jandel RMS-EL-Z.

\section{2. Экспериментальные результаты}

\section{1. Просвечивающая электронная микроскопия}

По данным ПЭМ толщина пленок, полученных при различной концентрации метана, примерно одинакова и составляет $30 \mathrm{~nm}$.

На рис. 1 показаны дифракционные картины, соответствующие пленкам, нанесенным в атмосфере чистого аргона $(a)$ и наибольшей концентрации метана $(b)$. Картины дифракции образцов, полученных при концентрации метана 6 и 33\%, схожи с представленной на рис. $1, b$.

На дифракционных картинах всех образцов присутствуют широкие кольца, отвечающие межплоскостным расстояниям 2.1 и $1.15 \AA$. Такие линии характерны для аморфных углеродных материалов [11] и свидетельствуют о структуре образцов типа ta-С. Для пленок, нанесенных в атмосфере с добавлением метана, в дифракционной картине появляется широкое гало, соответствующее межплоскостному расстоянию $3.8 \AA$ А. Наличие такого гало свидетельствует о появлении в структуре разупорядоченных нанокристаллических включений с бо́льшими, чем у графита $(3.35 \AA)$, межплоскостными расстояниями. Из литературных данных известно, что схожие межплоскостные расстояния свойственны цепочечным углеродным структурам. Так, характерное расстояние между цепочками для молекул линейно-цепочечного углерода, согласно экспериментальным данным [12], варьируется от 4 до $5 \AA$. Для цис-полиацетилена характерно межплоскостное расстояние $3.8-3.85 \AA$, для транс-полиацетилена $-3.68 \AA$ [13], а для полидиацетилена $-4.91 \AA[14]$.

На рис. 2 представлены изображения текстуры образцов.

Образец, полученный в атмосфере аргона, однороден (рис. $2, a)$. Пленки, изображенные на рис. $2, b-d$, содержат нанокристаллические включения, при этом в дифракционной картине наблюдается широкое гало, соответствующее межплоскостному расстоянию $3.8 \AA$. Характерный размер данных кластеров составляет $2-4 \mathrm{~nm}$. При низкой концентрации метана среднее расстояние между ними составляет $6-10 \mathrm{~nm}$ (рис. $1, b$ ). С увеличением доли метана в рабочем объеме концентрация включений существенно возрастает. При максимальном значении концентрации $52 \%$ кластеры образуют конгломераты размером $10-15 \mathrm{~nm}$ (рис. $2, d$ ).

Предположительно появление включений объясняется тем, что при увеличении концентрации метана перестройка структуры, связанная с разложением метана на подложке в процессе напыления углеродных пленок, происходит более эффективно.

\section{2. К КР-спектроскопия}

На рис. 3 представлены спектры комбинационного рассеяния образцов, полученных при различных концентрациях метана в атмосфере рабочей камеры. B спектрах пленок, нанесенных в атмосфере $\mathrm{Ar}$ без добавления $\mathrm{CH}_{4}$ (рис. $3-0 \%$ ), наблюдется широкий пик вблизи $1520 \mathrm{~cm}^{-1}$, характерный для аморфных углеродных структур типа ta-C [15]. Добавление метана приводит к росту интенсивности линий в диапазоне $2040-3150 \mathrm{~cm}^{-1}$, при этом форма основной линии с максимумом $1520 \mathrm{~cm}^{-1}$ остается практически неизменной.

В КР-спектре образца, полученного при наибольшей концентрации метана, наиболее ярко проявляется се- 

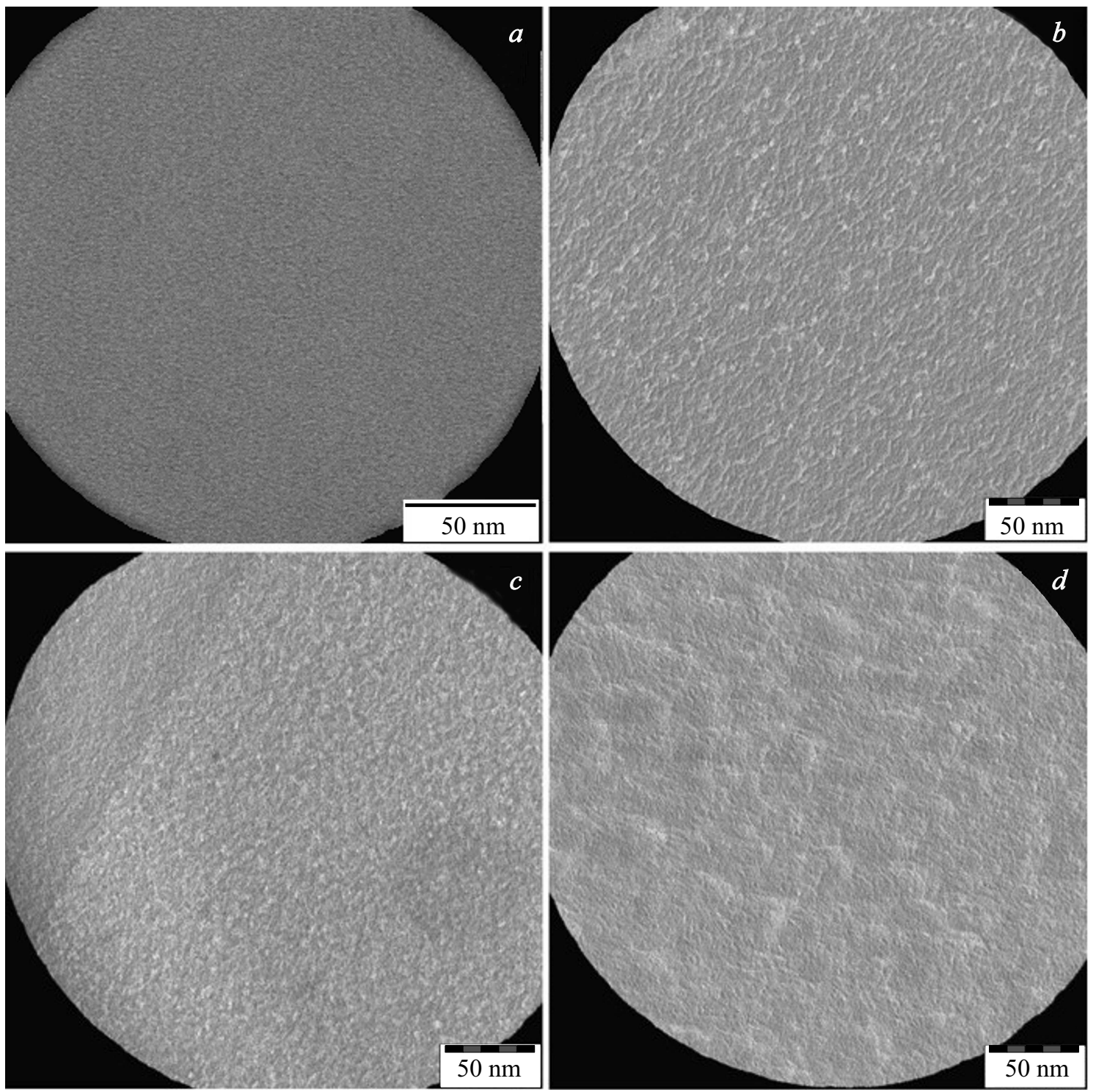

Рис. 2. Текстура образцов, напыленных при различной концентрации метана: $a-0, b-6, c-33, d-52 \%)$.

рия линий в диапазоне $2040-3150 \mathrm{~cm}^{-1}$. На рис. 4 представлено разложение данного спектра на гауссовы составляющие.

Пики, максимумы которых расположены на частотах $1310 \mathrm{~cm}^{-1}$ (D-линия) и $1520 \mathrm{~cm}^{-1}$ (G-линия), характерны для большинства углеродных структур. D-линия („disorder") свидетельствует о наличии дефектов или оборванных связей в исследуемой структуре, а G-линия (,graphite“) является признаком $s p^{2}$-гибридизованных атомов [16]. Положение, интенсивность и ширина данных линий могут использоваться для определения фазового состава аморфных образцов, содержащих $s p^{2}$ и $s p^{3}$-связи [17].

Линии 1100 и $2040 \mathrm{~cm}^{-1}$ соответствуют наличию в исследуемых пленках цепочек с $s p$-связями [18]. Как показано в работах $[19,20]$, наличие схожих линий в
КР-спектрах может объясняться образованием карбиноподобной структуры.

Постоянство положения и относительных интенсивностей D- и G-линий свидетельствует о неизменности структуры аморфной компоненты пленки. Таким образом, рост линии $2040 \mathrm{~cm}^{-1}$ при увеличении доли метана в камере можно интерпретировать как увеличение доли $s p$-содержащих включений в аморфной $s p^{2} / s p^{3}$-фазе. Полученные данные хорошо коррелируют с результатами ПЭМ, показывающими появление фазы с большими межплоскостными расстояниями, возрастание концентрации и агломерацию нанокристаллических включений при увеличении доли метана.

Особенностью спектров образцов, полученных при добавлении в атмосферу рабочей камеры метана, является серия квазипериодичных линий, лежащих в диа- 
пазоне 2040-3000 $\mathrm{cm}^{-1}$. Согласно нашим предположениям, данные линии можно интерпретировать как многофононные реплики линии $2040 \mathrm{~cm}^{-1}$. Явление многофононных реплик наблюдается в спектрах люминесценции $[21,22]$, зависимости фототока от энергии возбуждающего фотона [23], в ИК- и КР-спектрах различных структур [24,25]. Согласно данной интерпретации, сателлиты основной линии появляются, когда фотон, соответствующий ей, затрачивает энергию на возбуждение одного или нескольких фононов, смещаясь в спектре дальше от основной линии на величину, соответствующую энергии фонона.

Анализ спектра показывает, что расстояние между линиями для исследованных образцов составляет примерно $210 \mathrm{~cm}^{-1}$. Это значение по порядку величины соответствует интервалам между сериями максимумов,

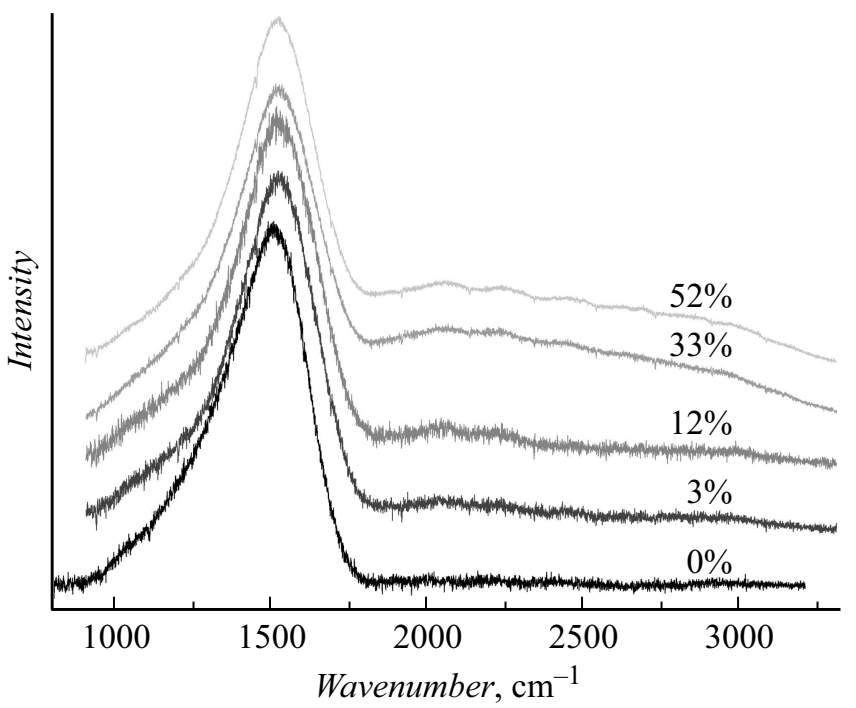

Рис. 3. КР-спектры образцов, напыленных при различной концентрации метана.

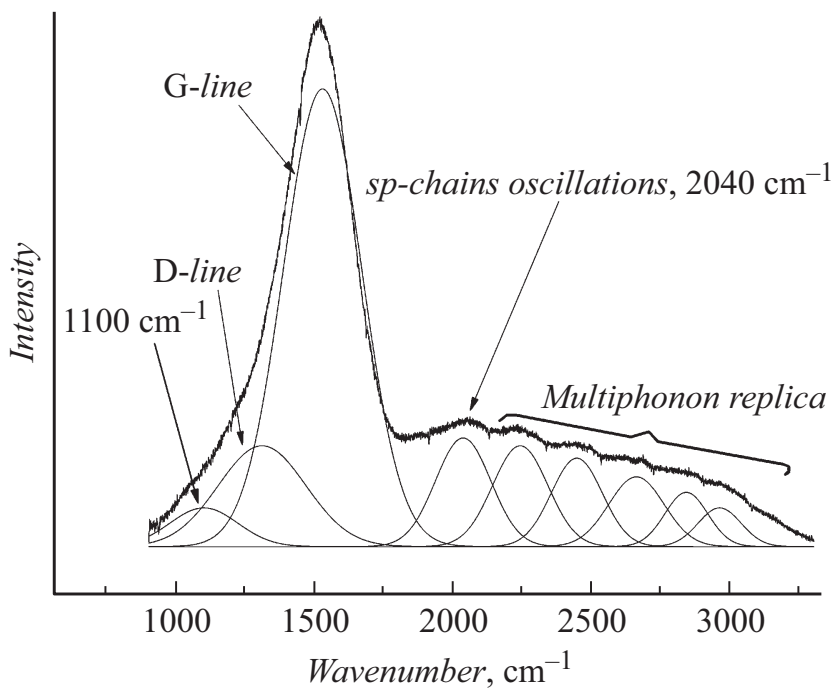

Рис. 4. КР-спектр образца, напыленного при концентрации метана $52 \%$.

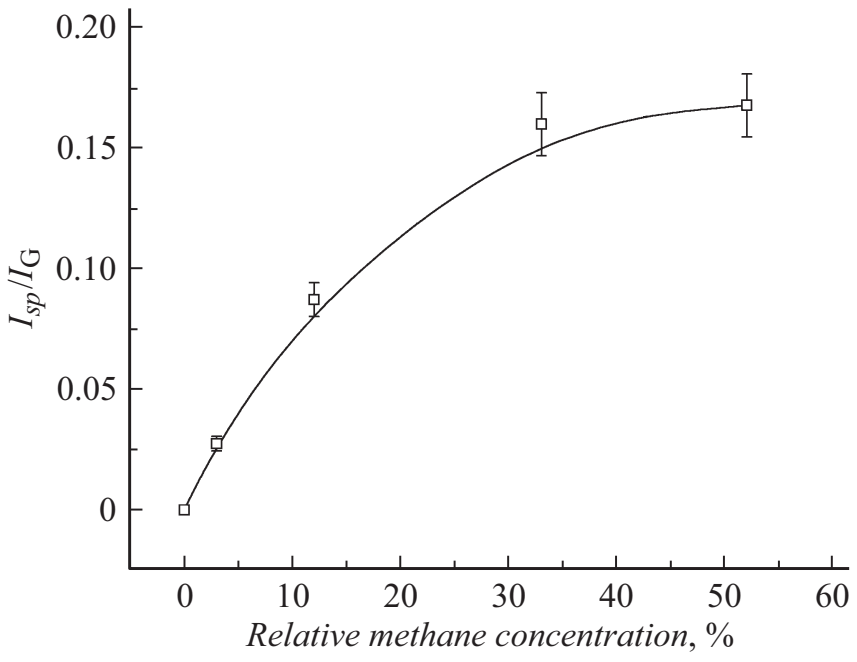

Рис. 5. Зависимость соотношения интенсивностей линии с максимумом $2040 \mathrm{~cm}^{-1}$ и G-линии от концентрации метана в камере.

наблюдаемых в спектрах люминесценции и ИК-спектрах углеродных структур, где расстояние между репликами варьируется от 120 до $810 \mathrm{~cm}^{-1}$ [26-29].

C ростом концентрации метана увеличивается соотношение интенсивности линий 2040 и $1520 \mathrm{~cm}^{-1}$, т.е. возрастает доля $s p$-гибридизированного углерода в аморфной фазе ta-C-структуры.

На рис. 5 представлена зависимость отношения интенсивности максимума, связанного с углеродными цепочками с $s p$-гибридизацией $\left(2040 \mathrm{~cm}^{-1}\right)$, к пику, соответствующему наличию графитовых связей в структуре (G-линия), от концентрации метана в рабочей атмосфере вакуумной камеры. С увеличением концентрации метана данное соотношение растет, что свидетельствует об увеличении доли $s p$-гибридизированного углерода в структуре.

\section{3. Удельное электросопротивление}

Зависимость удельной электропроводности исследуемых образцов от концентрации метана представлена на рис. 6. Как видно из графика, при введении метана удельное сопротивление возрастает на несколько порядков, причем данная зависимость хорошо коррелирует с описанной выше зависимостью отношения интенсивностей $I_{s p} / I_{\mathrm{G}}$ от концентрации метана в рабочем объеме, что свидетельствует об определяющем вкладе $s p$-гибридизованного углерода в электропроводность всей структуры. Это также согласуется с высокими значениями удельного сопротивления, характерными для карбиноподобных материалов [30].

Влияние $s p$-компоненты на электрофизические свойства структуры и на их КР-спектры было описано для аморфных пленок, состоящих из $s p$ - и $s p^{2}$-фаз, в работе [31], где увеличение температуры подложки при- 


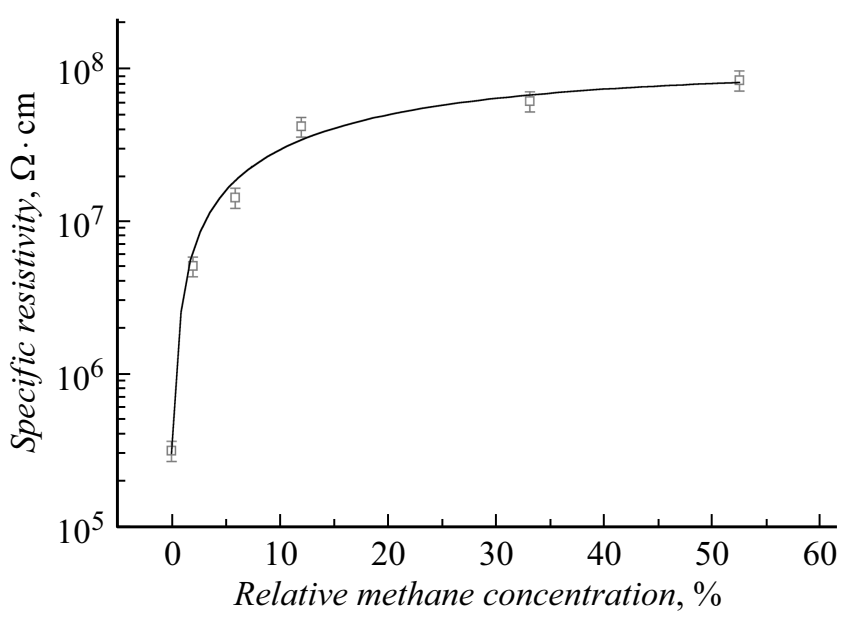

Рис. 6. Зависимость удельного сопротивления от относительной концентрации метана в камере

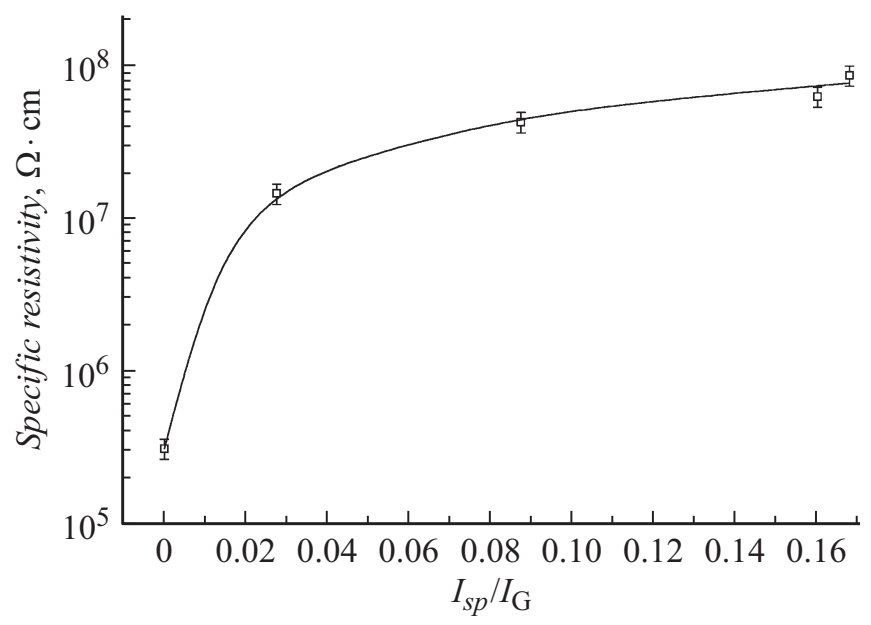

Рис. 7. Зависимость удельного электросопротивления образцов от соотношения линии $2040 \mathrm{~cm}^{-1}$ и G-линии КР-спектра.

водило к уменьшению $s$-фазы в структуре пленок и увеличению их проводимости. В свою очередь, для ta-C-покрытий, получаемых в среде метана и аргона при помощи магнетронного распыления графитовой мишени, было описано увеличение доли $s p^{3}$-гибридизованных атомов по отношению к $s p^{2}$-гибридизованным при увеличении концентрации метана, приводящее к росту удельного электросопротивления [32].

В отличие от приведенных выше исследований, в настоящей работе при ионно-плазменном импульснодуговом распылении в атмосфере метана и аргона происходит увеличение доли $s p$-фазы в общей аморфной матрице углеродной пленки. При этом, согласно данным ПЭМ, доля $s p$-фазы возрастает за счет образования $s p$-содержащих кластеров с большими межцепочечными расстояниями в $s p^{2} / s p^{3}$-аморфной фазе, что приводит к увеличению сопротивления структуры. Рис. 7 показывает корреляцию роста интенсивности линии $2040 \mathrm{~cm}^{-1}$ и удельного электросопротивления.
Увеличение доли $s p$-фазы в углеродных пленках при возрастании концентрации метана предположительно объясняется эффективным разложением молекул метана на поверхности образца под действием импульсного разряда. Образование сильных цепочечных связей в ходе этого процесса приводит к образованию кластеров, характеризующихся большим межплоскостным расстоянием, что свидетельствует об их преимущественной $s p$-гибридизации.

\section{Заключение}

В работе методом импульсно-плазменного дугового распыления графита были получены углеродные покрытия с различной концентрацией $s p$-фазы в структуре аморфной пленки типа ta-C. Исследования структуры и фазового состава показали, что увеличение концентрации метана в атмосфере рабочей камеры приводит к увеличению числа $s p$-гибридизованных нанокластеров и их агломерации. Данное изменение структуры пленки приводит к увеличению ее удельного сопротивления на два порядка, до $10^{8} \Omega \cdot \mathrm{cm}$.

\section{Финансирование работы}

Работа была выполнена при финансовой поддержке Министерства образования и науки Российской Федерации в рамках выполнения соглашения № 14.625.21.0041 от 26.09.2017г. (уникальный идентификатор прикладных научных исследований RFMEFI62517X0041).

\section{Конфликт интересов}

Авторы заявляют, что у них нет конфликта интересов.

\section{Список литературы}

[1] Chhowalla M., Ferrari A. C., Robertson J., Amaratunga G.A.J. // Appl. Phys. Lett. 2000. Vol. 76. N 11. P. 14191421. doi: $10.1063 / 1.126050$

[2] Zhang T.F., Deng Q.Y., Liu B., Wu B.J. // Surf. Coat. Technol. 2015. Vol. 273. P. 12-19. https://doi.org/10.1016/j.surfcoat.2015.03.031

[3] Santra T.S., Bhattacharyya T.K., Patel P., Tseng F.G., Barik T.K. Microelectromechanical Systems and Devices. InTech. 2012. 480 p. DOI: 10.5772/29671

[4] Moers J., Trellenkamp S., Goryll M., Marso M., van der Hart A., Hogg S. et al. // Microelectronic Engineering. 2002. Vol. 64. N 1-4. P. 465-471. doi: 10.1016/s0167- 9317(02)00822-55)

[5] Grill A. // Thin Solid Films. 1999. Vol. 355-356. P. 189-193. https://doi.org/10.1016/S0040- 6090(99)00516-7

[6] Allen M.T., Shtanko O., Fulga I.C., Akhmerov A., Watanabi K., Taniguchi T., Jarillo-Herrero P., Levitov L.S., Yacoby A. // Nat. Phys. 2016. Vol. 12. P. 128-133. doi: $10.1038 /$ nphys3534

[7] Banszerus L., Schmitz M., Engels S., Goldsche M., Watanabe K., Taniguchi T. et al. // Nano Lett. 2016. Vol. 16. N 2. P. 1387-1391. doi: $10.1021 /$ acs.nanolett.5b04840 
[8] Casari C.S., Milani A. // MRS Communications. 2018. Vol. 8. N 02. P. 207-219. doi: $10.1557 / \mathrm{mrc} .2018 .48$

[9] Cretu O., Botello-Mendez A.R., Janowska I., Pham-Huu C., Charlier J.-C., Banhart F. // Nano Letters. 2013. Vol. 13. N 8. P. 3487-3493. doi: 10.1021/n14018918

[10] Ben Romdhane F., Adjizian J.-J., Charlier J.-C., Banhart F. // Carbon. 2017. Vol. 122. P. 92-97. doi: 10.1016/j.carbon.2017.06.039

[11] Liu Y., Meletis E. // J. Mater. Sci. 1997. Vol. 32. P. 3491-3495. https://doi.org/10.1023/A:1018641304944

[12] Бабаев В., Гусева М., Новиков Н., Савченко Н., Хвостов B. // Нанотехнологии: разработка, применение. 2010. T. 2. № 1. C. 88-94.

[13] Chien J.C.W. Polyacetylene. Chemistry, Physics, and Material Science. Orlando, Florida: Academic Press, 1984. 648 p.

[14] Enkelmann V. // Advanc. Polymer Sci. 1984. P. 91-136. doi: $10.1007 / \mathrm{bfb} 0017652$

[15] Ferrari A., Robertson J. // Phil. Trans. R. Soc. Lond. A. 2004. Vol. 362. P. 2477-2512. DOI: 10.1098/rsta.2004.1452

[16] Pimenta M.A., Dresselhaus G., Dresselhaus M.S., Cançado L.G., Jorio A., Saito R. // Phys. Chem. Chem. Phys. 2007. Vol. 9. N 11. P. 1276-1290. doi: 10.1039/b613962k

[17] Shchegolikhin A., Lazareva O. // Biomolecular Spectroscopy. 1997. Vol. 53. P. 67-79. doi: 10.1016/s1386-1425(97)83010-7

[18] Shi L., Rohringer P., Suenaga K. // Nature Materials. 2016. P. 634-640. DOI: 10.1038

[19] Milani A., Lucotti A., Russo V. // J. Phys. Chem. C. 2011. Vol. 115. P. 12836-12843. DOI: $10.1021 /$ jp203682c

[20] Lucotti A., Tommasini M., Del Zoppo M. // Chem. Phys. Lett. 2006. Vol. 417. P. 78-82. https://doi.org/10.1016/j.cplett.2005.10.016

[21] Teke A., Özgür Ü., Doğan S., Gu X., Morkoc H., Nemeth B., Nause J., Everitt H.O. // Phys. Rev. B. 2004. Vol. 70. P. 195207. DOI: 10.1103/PhysRevB.70.195207

[22] Blanks D.K., Bicknell R.N., GilesTaylor N.C., Schetzina J.F., Petrou A., Warnock J. // J. Vacuum Sci. Technol. 1986. A 4. P. 2120. http://avs.scitation.org/doi/10.1116/1.574040

[23] Tsukada N., Kikuta T., Ishida K. // Phys. Rev. 1986. Vol. 3. N 12. P. 8859-8862.

[24] Goasa K., Grzeszczyk M., Leszczyński P., Faugeras C., Nicolet A.A.L., Wysmotek A., Potemski M., Babiński A. // Appl. Phys. Lett. 2014. Vol. 104. P. 092106. http://aip.scitation.org/doi/abs/10.1063/1.4867502

[25] Manasreh M.O., Covington B.C. // Phys. Rev. B. 1987. Vol. 35. N 5. https://journals.aps.org/prb/abstract/10.1103/ Phys.Rev.B.35.2524.

[26] Collins A.T., Lawson S.C., Davies G., Kanda H. // Phys. Rev. Lett. 1990. Vol. 65. N 7. P. 891-894. doi:10.1103/physrevlett.65.891

[27] Watanabe H., Hayashi K., Takeuchi D., Yamanaka S., Okushi H., Kajimura K., Sekiguchi T. Appl. Phys. Lett. 1998. Vol. 73. N 7. P. 981-983. doi: $10.1063 / 1.122059$

[28] Weima J.A., Zaitsev A.M., Job R., Kosaca G.C., Blum F., Grabosch G., Knopp J. // IECON'99. Conf. Proc. 25th Annual Conf. of the IEEE Industrial Electronics Society (Cat. N 99CH37029). doi: 10.1109/iecon.1999.822170

[29] Bazhenov A.V., Kveder V.V., Maksimov A.A., Tartakovskii I.I., Oganyan R.A., Ossipyan Y.A., Shalynin A.I. // J. Experiment. Theoret. Phys. 1998. Vol. 86. N 5. P. 1030-1034. doi: $10.1134 / 1.558550$

[30] Brazhkin V.V., Lyapin A.G., Popova S.V. // JETP Lett. 2002. Vol. 76. N 11. P. 681-692. https://doi.org/10.1134/1.1545583

[31] Ravagnan L., Piseri P., Bruzzi M., Miglio S., Bongiorno G., Baserga A. // Phys. Rev. Lett. 2007. Vol. 98. N 21. doi: $10.1103 /$ physrevlett.98.216103

[32] Mansano R.D., Massi M., Verdonck P., Nogueira P.M. // Superficies y Vacío. 1999. Vol. 9. P. 111-114. 